\title{
Modelling of self-aligned total internal reflection waveguide mirrors: an interlaboratory comparison
}

\section{J. ČTYROKÝ}

Institute of Radio Engineering and Electronics, Academy of Sciences of the Czech Republic, Chaberská 57, 18251 Praha 8, Czech Republic

H. HOEKSTRA, G. J. M. KRIJNEN, P. V. LAMBECK University of Twente, MESA Research Institute, Enschede, The Netherlands

L. JOANNES, D. DECOSTER Institute d'Electronique et Microélectronique du Nord, Villeneuve d'Ascq, France

A. P. LEITE, M. ADELAIDE ANDRADE University of Porto, Faculty of Sciences, Porto, Portugal

R. PREGLA, E. AHLERS

University for Distant Studies, Hagen, Germany

J.-F. VINCHANT

Alcatel Alsthom Recherche, Marcoussis, France

(Now with Alcatel Optronics, Device \& Technology Department, Nozay Cedex, France)

Received 12 December 1994; revised 19 April; accepted 3 May 1995

Results of modelling of light propagation in $45^{\circ}$ self-aligned total internal reflection rib waveguide mirrors on InP substrate are compared. Six laboratories participated in the comparison with the following six modelling methods: the standard fast-Fouriertransform beam propagation method (BPM), the standard finite-difference (FD) BPM using the Crank-Nicholson scheme (two laboratories), the FD-BPM with the correction for the slowly varying envelope approximation, the method of lines, the eigenmode expansion and propagation method, and a simple method based on the field overlap. All the laboratories used the effective-index method to reduce the three-dimensional problem to two dimensions. The differences among the results obtained by different methods are briefly discussed and qualitatively compared to measured values.

\section{Introduction}

Self-aligned waveguide total internal reflection (TIR) mirrors are becoming important building blocks of modern photonic integrated circuits (PIC) based on semiconductor substrates. Using TIR mirrors, it is possible to realize abrupt changes of the direction of the waveguides without 


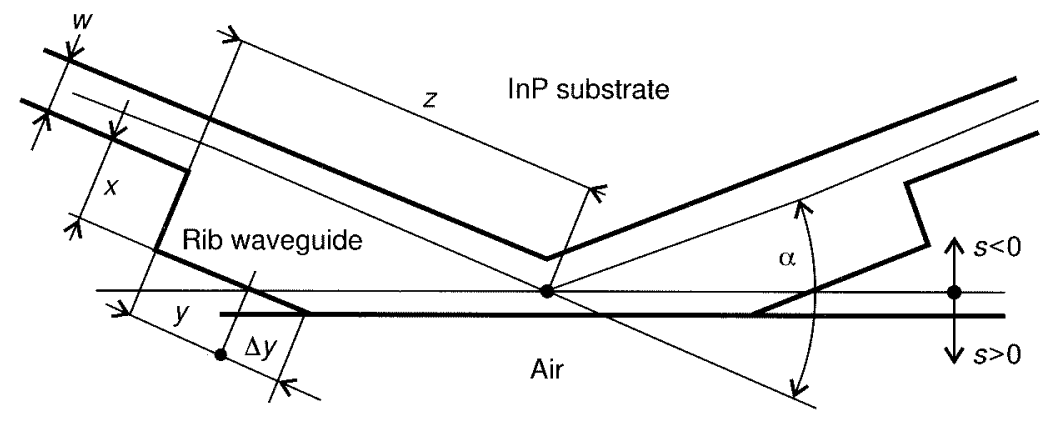

Figure 1 Geometry of the self-aligned waveguide TIR mirror.

wasting substrate length and/or surface area compared to conventional bends. This property would make TIR mirrors indispensable in designing complex PICs with large scale of integration if their loss were low enough. It has been shown by several laboratories (HHI Berlin [1], AAR Marcoussis [2], ETH Zurich and others) that the reflection loss of $45^{\circ}$ and $90^{\circ}$ waveguide mirrors can be as low as a few tenths of a $\mathrm{dB}$ per facet. This value has also been confirmed by a recent round-robin test measurement [3] performed within the framework of the COST 240 Project 'Techniques of Modelling and Measuring Advanced Photonic Telecommunication Components'.

The aim of the work reported here was to compare results of modelling of the TIR ribwaveguide mirror by different methods that have recently been developed and are now currently used in various research laboratories. The task was to calculate the dependence of reflectivity of an InP rib-waveguide TIR mirror on the offset of the reflecting surface from the geometric-optical mirror position, for several rib waveguide widths. The calculated optimum mirror position and the magnitude of the minimum reflection loss were of special interest. The results of calculations are also qualitatively compared to loss values obtained experimentally.

\section{Modelling task}

A schematic 'top view' of the modelled waveguide mirror with total internal reflection is shown in Fig. 1. The waveguide deflection angle $\alpha$ was $45^{\circ}$. Losses of the fundamental mode of the waveguide were to be calculated for several values of the offset $s$, namely for $s=-0.4 w$, $-0.3 w, \ldots, 0.3 w, 0.4 w$, for waveguide widths $w=4,5$ and $6 \mu \mathrm{m}$.

As seen from Fig. 1, the waveguide is stepwise broadened by $x=5 \mu \mathrm{m}$ in the vicinity of the mirror plane. This broadening is for purely technological reasons, namely to enlarge the mirror edge length in order to ease the mask alignment. From simple geometrical considerations, the

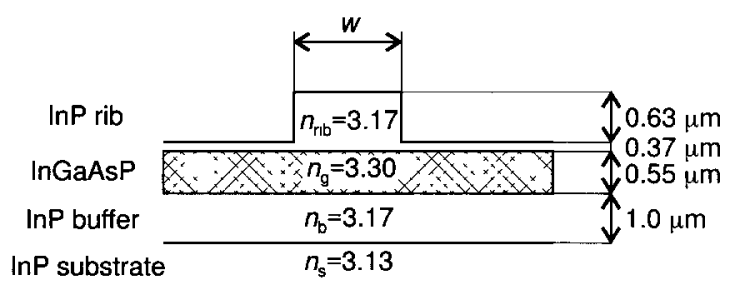

Figure 2 Rib waveguide cross-section with refractive indices of the layers. 


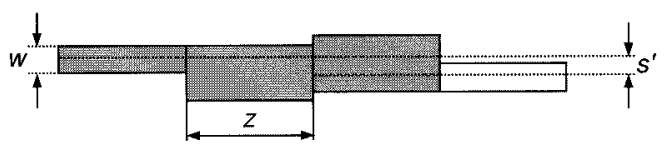

Figure 3 'Effective' waveguide structure used in the field overlap (A-MEP) method.

following expressions can be found for the dimensions $y, \Delta y$ and $z$ defined in Fig. 1:

$$
y=\frac{w}{\sin \alpha}+x \sin (\alpha / 2) \quad z=y+\frac{x+w / 2}{\tan (\alpha / 2)} \quad \Delta y=\frac{s}{\sin (\alpha / 2)}
$$

The transverse structure of the modelled rib waveguide is shown in Fig. 2. It is adapted partly from waveguide structures developed in Alcatel Alsthom Recherche in Marcoussis, and on ETH Zurich. The refractive indices shown in the figure correspond approximately to the wavelength of $1.52 \mu \mathrm{m}$. The calculations were to be made for both (quasi) TE and TM polarizations. The choice of the modelling method was completely free.

\section{Overview of modelling methods}

All contributors from the participating laboratories decided to reduce the rather complicated 3D problem with strongly off-axis propagation and very large refractive-index contrast to a simpler 2D one by the effective-index method (EIM). Five different 'beam propagation' methods were used as modelling tools, namely the classical BPM based on the fast-Fourier-transform algorithm (FFT-BPM) [4], the standard FD-BPM method based on paraxial approximation and Crank-Nicholson scheme [5] with properly chosen reference refractive index (two independent codes), the 'enhanced' FD-BPM including second-order correction for the slowly-varying envelope approximation (E-FD-BPM) [6], the BPM algorithm based on the method of lines (MoL-BPM) [7], and the unidirectional option of the bidirectional eigenmode expansion and propagation method [8] using transverse discretization by the finite-difference method (FD-MEP) [9]. Additionally, a method based on the field overlap calculation using the analytical MEP (A-MEP) similar to [8] was also used for comparison. The latter method takes into account the Goos-Hänchen shift and the offset of the mirror but not the change of the modal field distribution by total reflection. Instead of the waveguide structure in Fig. 1, this method modelled the 'equivalent waveguide structure' shown in Fig. 3. The 'effective shift' $s^{\prime}$ takes into account the Goos-Hänchen shift by total reflection at the waveguide-air interface. For its calculation, the effective refractive index of the mode was taken as the refractive index of the optically denser medium.

The methods used in the test are listed in Table I, together with the laboratories where they

TABLE I Methods, participating laboratories, size of discretization steps used, and polarization calculated

\begin{tabular}{llll}
\hline Method & Laboratory & Step size $\Delta x \times \Delta z(\mu \mathrm{m})$ & Polarization \\
\hline FFT-BPM & AAR Marcoussis & $0.125 \times 0.125$ & TE, TM \\
FD-BPM & IEMN Villeneuve d'Ascq & $0.01 \times 0.2$ & TM \\
FD-BPM & University of Porto & $0.01 \times 0.05$ & TE, TM \\
E-FD-BPM & MESA Uni Twente & $0.03 \times 0.1$ & TE \\
Mol BPM & Fern-Uni Hagen & $0.05 \times 0.12$ & TE \\
FD-MEP & IREE Prague & $0.06 \times 0.2$ & TE, TM \\
Overlap by A-MEP & IREE Prague & No discretization & TE, TM \\
\hline
\end{tabular}




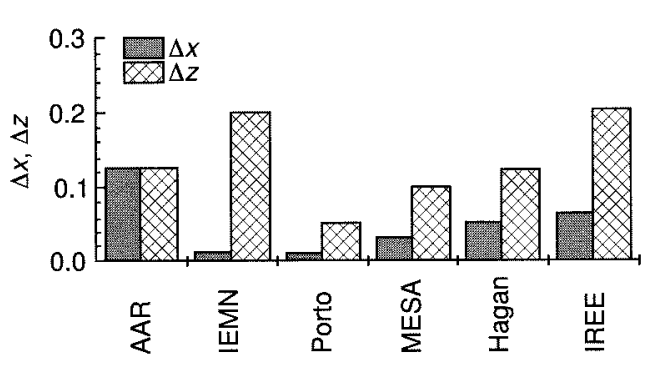

Figure 4 Sizes of discretization steps used by the participating laboratories for the different methods (Hagen 4 corresponds to $\mathrm{MoL}$ for $w=4 \mu \mathrm{m}$ ).

were developed. Also shown are the sizes of discretization steps in both transverse and longitudinal directions and the polarization for which the calculation was done. The sizes of discretization steps are plotted in Fig. 4. In all of the methods but the FFT-BPM, the mode field overlap calculation of the power carried by an individual mode of the waveguide is implemented, while in the FFT-BPM method, the total power propagating in the waveguide is calculated by the light intensity integration over the waveguide 'core'.

\section{Results and discussion}

Results corresponding to (quasi) TE modes were available from five of the listed contributing
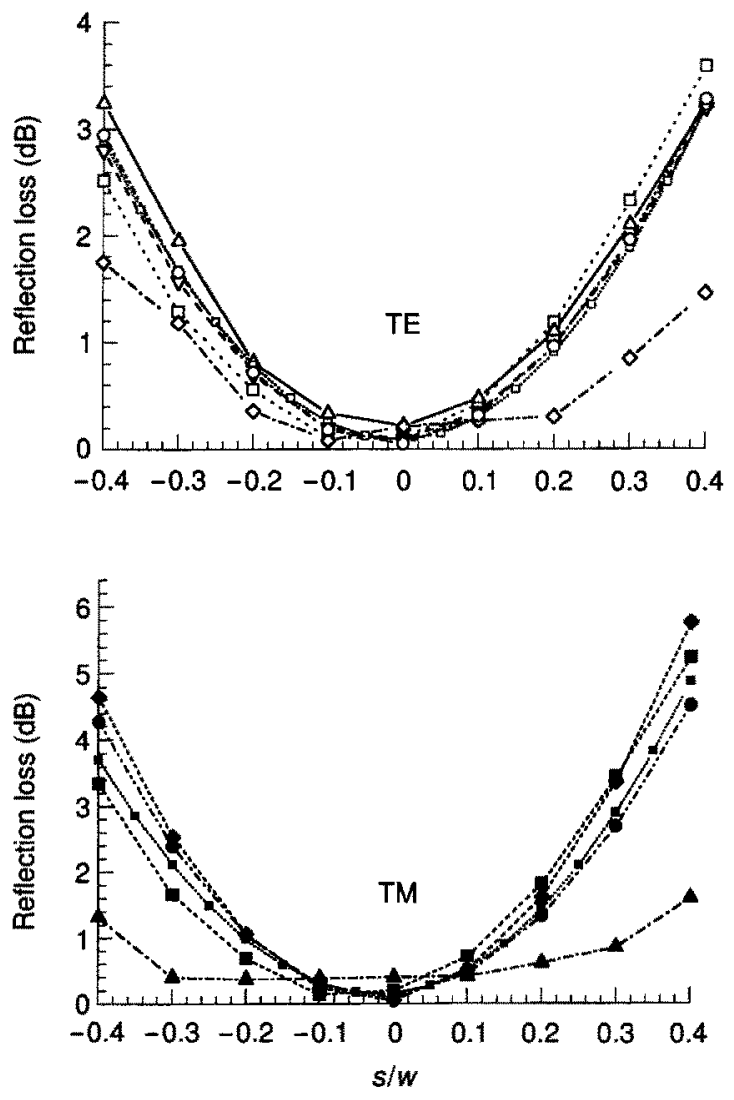

Figure 5 Calculated dependence of the $45^{\circ}$ TIR mirror loss on the position of the reflecting surface; waveguide width $4 \mu \mathrm{m}$. TE polarization: $\diamond$, FFT-BPM, AAR Marcoussis; O, FD-BPM, Uni Porto; $\nabla$, E-FD-BPM, MESA Twente; $\triangle$, MoL, Fern-Uni Hagen; $\square$, FD-MEP, IREE Prague; $\square$, overlap integral by A-MEP. TM polarization: $A$, FFTBPM, AAR Marcoussis; $\$$, FD-BPM, IEMN Villeneuve; 9 , FD-BPM, Uni Porto; E, FDMEP, IREE Prague; a. overlap integral by A-MEP. 

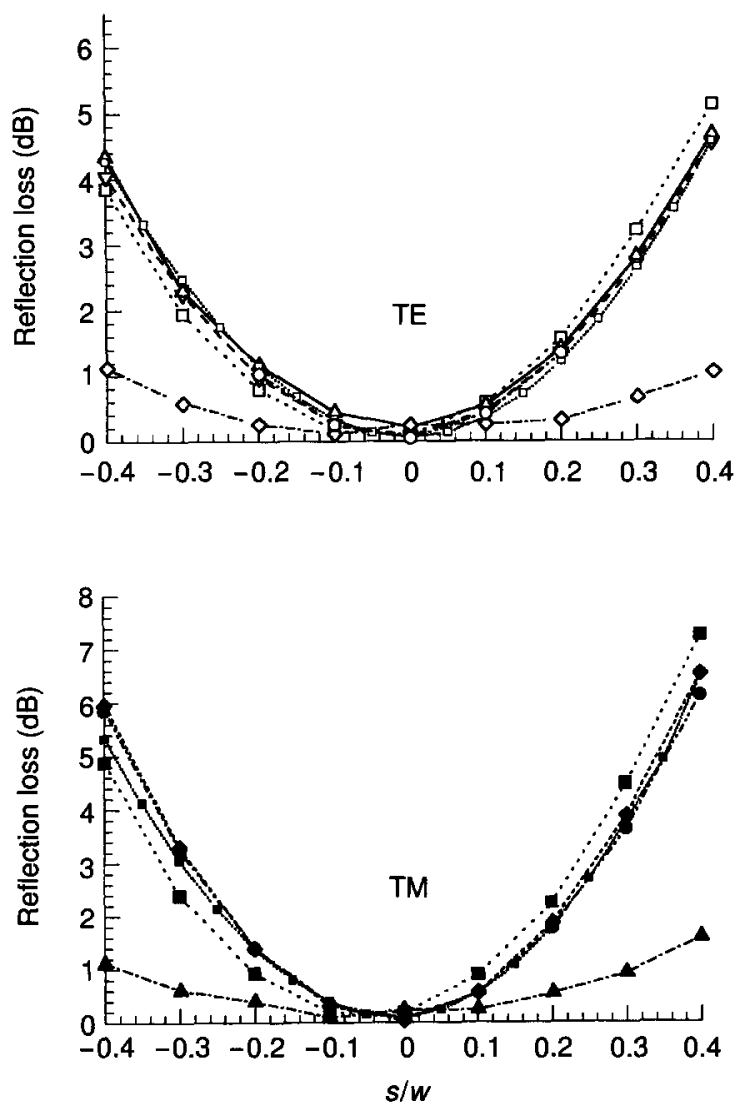

Figure 6 Same as in Fig. 5 but for waveguide width $5 \mu \mathrm{m}$. TE polarization: $\diamond$, FFTBPM, AAR Marcoussis; O, FD-BPM, Uni Porto; $\nabla$, E-FD-BPM, MESA Twente; $\triangle$, MoL, Fern-Uni Hagen; $\square$, FD-MEP, IREE Prague; $\square$, overlap integral by A-MEP. TM polarization: $\triangle$, FFT-BPM, AAR Marcoussis; $\bullet$, FD-BPM, IEMN Villeneuve; 9 , FDBPM, Uni Porto; $\square$, FD-MEP, IREE Prague; $\square$, overlap integral by A-MEP.

laboratories by six methods, and four of them also calculated the TM case. The results are collected in Figs 5 to 7 for the widths 4 to $6 \mu \mathrm{m}$, respectively.

For the purposes of comparison, the case of $w=4 \mu \mathrm{m}$ and TE polarization is re-plotted in Fig. 8, relative to the losses calculated by the field overlap method (A-MEP in Table I), i.e. with subtracted A-MEP values.

The spread in the results delivered by different methods is quite significant, especially for narrower waveguides and TE polarization, but since there is not a priori a known exact result, the accuracy of the individual methods cannot be judged reliably. The results of the FFT-BPM differ most significantly from all other methods. As this feature is more pronounced for wider waveguides that are not purely laterally single-mode (Fig. 7), we tend to the belief that the main reason for this large difference lies in the way the power in the waveguide was calculated. The lateral (effective) index contrast in the waveguide is very small everywhere except in the mirror region. The $\mathrm{FD}, \mathrm{MoL}$ and MEP methods agree quite well around the loss minimum for $w=4 \mu \mathrm{m}$. Most methods found the smallest losses for the mirror position close to the optimum determined from the mirror reflection with Goos-Hänchen shift taken into account. (This optimum mirror position is very close to the geometric-optical mirror position, with $s \cong-0.08 \mu \mathrm{m}$ for TM modes and even less for TE modes, so that in the practical design of the mirror this offset can be neglected.) The generally very good agreement of the FD-BPM and Mol-BPM methods with the A-MEP method implies that technologically important 

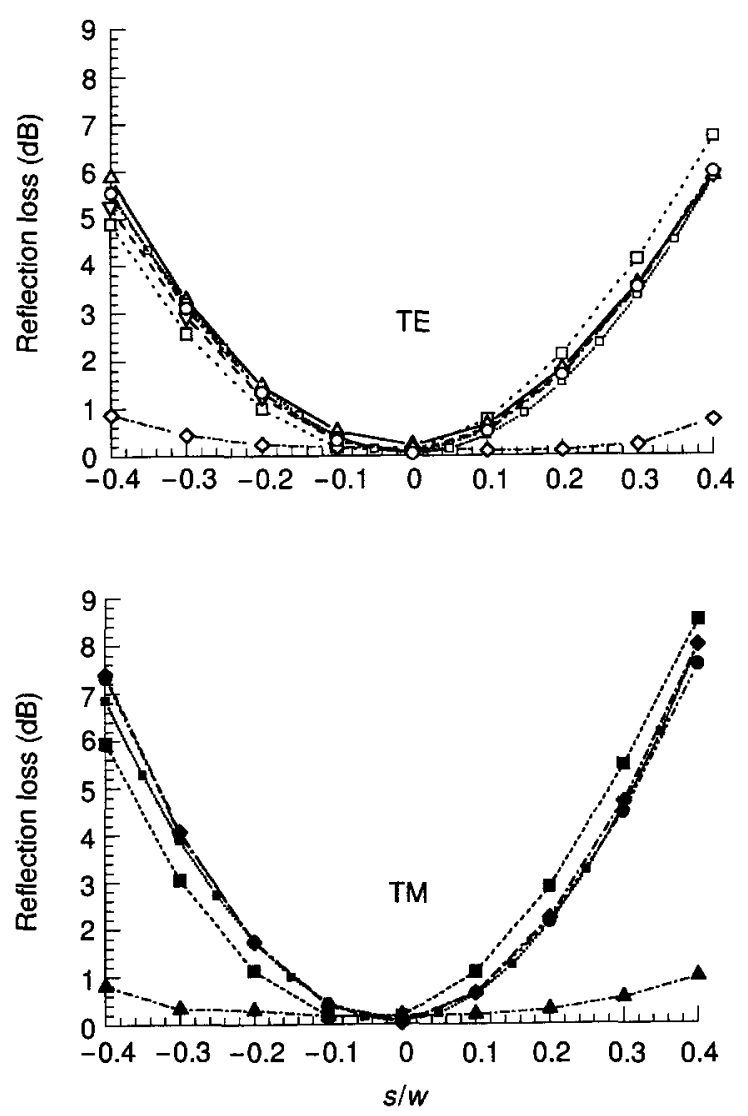

Figure 7 Same as in Fig. 5 but for waveguide width $6 \mu \mathrm{m}$. TE polarization: $\diamond$, FFTBPM, AAR Marcoussis; O, FD-BPM, Uni Porto; $\nabla$, E-FD-BPM, MESA Twente; $\triangle$. MoL, Fern-Uni Hagen; $\square$, FD-MEP, IREE Prague; $\square$, overlap integral by A-MEP. TM polarization: $\triangle$, FFT-BPM, AAR Marcoussis; , FD-BPM, IEMN Villeneuve; $\bigcirc$, FDBPM, Uni Porto; $\square$, FD-MEP, IREE Prague; $\boldsymbol{D}$, overlap integral by A-MEP.

parameters - the optimum position of the mirror plane and the loss behaviour as a function of the offset - can be well understood using simple physical considerations. These methods give the most consistent results, perhaps because of their small discretization steps (see the last column in Table I and Fig. 4). Good applicability of the standard FD-BPM based on the Fresnel approximation should not be surprising. In the mirror, the wave energy propagates mostly

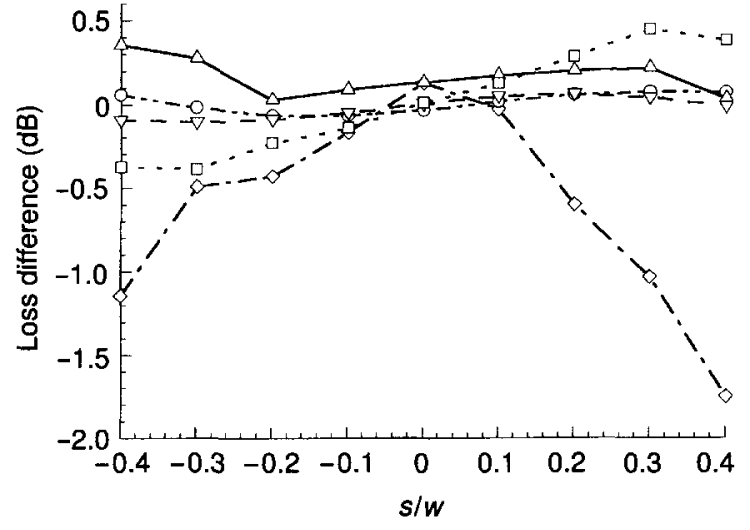

Figure 8 Difference among the 'BPM' methods for $w=4 \mu \mathrm{m}$ and TE polarization, with field overlap method (A-MEP) as a 'reference'. $\diamond$, FFT-BPM, AAR Marcoussis; $O$, FD-BPM, Uni Porto; $\nabla$, E-FD-BPM, MESA Twente; $\triangle$, MoL, Fern-Uni Hagen; $\square$, FD-MEP, IREE Prague. 


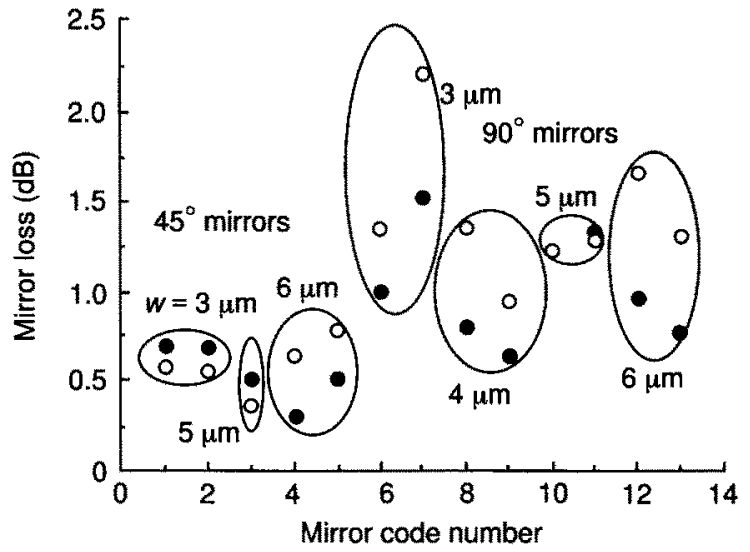

Figure 9 Averaged experimental losses of $45^{\circ}$ and $90^{\circ}$ TIR mirrors on InP rib waveguides 3,4 and $6 \mu \mathrm{m}$ wide (adapted from [3]). $O$, TE modes; $O$, TM modes.

inside a narrow angle around $\pm 22.5^{\circ}$ that can be well covered by the method if the reference refractive index is properly chosen. In contrast, the E-FD-BPM was proven to be insensitive to large variations of the reference refractive index. Most methods give a minimum loss value slightly less than $0.1 \mathrm{~dB}$. This is in reasonable agreement with measured data shown in Fig. 9 if we take into account that the EIM reduction to a $2 \mathrm{D}$ problem generally tends to underestimate the radiation losses, and moreover that scattering losses and nonverticality of the real mirrors as additional sources of losses are neglected in the model.

The losses in Fig. 9 are averaged from results obtained in the round-robin test [3] on InP waveguide TIR mirrors of similar structure.

\section{Conclusions}

Note first that the comparative calculations clearly confirmed our experience that it is not trivial to get reliable results applying even a well-tested and currently used (BPM) modelling method to a novel problem. Reflection from a self-aligned waveguide TIR mirror was modelled by six different 'BPM' techniques and compared to a simple field overlap method. The overlap integral method gives valuable insight into the behaviour of the mirror with much less computational effort. A minimum loss of about $0.1 \mathrm{~dB}$ was found by most of the methods for the optimum reflector position of the $45^{\circ}$ mirror. The optimum position differs only very little from the geometric-optical reflection plane and is well predicted by the Goos-Hänchen shift. As all the methods use the EIM reduction of the problem to the simpler 'planar' case, the inaccuracy introduced by EIM could not be distinguished. In view of this uncertainty and of unavoidable technological imperfections of real mirrors, the agreement between measured and calculated values of reflection losses is rather good. It was not possible to select unanimously the most reliable method. Instead, it was observed that the deviations of the results from those obtained by the mode overlap method are attributable to the size of the discretization steps used for the calculation rather than to the method itself. From this we may conclude that all the methods used in the comparison have the potential to possess results reliably in the EIM approximation.

\section{Acknowledgements}

This work was done in the framework for the EU COST 240 Project 'Techniques of Modelling and Measuring Advanced Photonic Telecommunication Components'. The authors thank E. Gini for detailed results of measurements reported in [3]. 


\section{References}

1. P. ALBRECHT, W. DÖLDISSEN, U. NIGGEBRUGGGE, H. P. NOLTING and H. SCHMIDT, ECOC '87, Helsinki, 1987, p. 239.

2. J.F. VINCHANT, Proc. COST Workshop 94: Optical Telecommunications Fibres and Components for System Applications: Present and Future, Nice, 1994, p. 82.

3. E. GINI, L. H. SPIEKMAN, H. VAN BRUG et al, Technical Digest, Symposium on Optical Fiber Measurements, Boulder, 1994, p. 113.

4. M. D. FEIT and J. A. FLECK, Appl. Opt. 17 (1978) 3390.

5. Y. CHUNG and N. DAGLI, IEEE J. Quantum Electron. 26 (1990) 1335.

6. H. J. W. M. HOEKSTRA, G. J. M. KRINEN and P. V. LAMBECK, Opt. Commun. 17 (1993) 301.

7. J. GERDES and R. PREGLA, J. Opt. Soc. Am. B 8 (1991) 389.

8. G. SZTEFKA and H.-P. NOLTING, IEEE Photon. Technol. Lett. 5 (1993) 554.

9. J. C̆TYROKÝ and 1. PLEŠINGR, SPIE Conf. 2150: Fabrication, Design and Modelling of Optical Guded-Wave Structures and Devices, Los Angeles, 1994, p. 116. 